\title{
Alpha and Beta Band Event-Related Desynchronization Reflects Kinematic Regularities
}

\author{
Yaron Meirovitch, ${ }^{1 \star}$ Hila Harris, ${ }^{2 \star}$ Eran Dayan, ${ }^{3}$ Amos Arieli, ${ }^{2}$ and Tamar Flash ${ }^{1}$ \\ ${ }^{1}$ Department of Computer Science and Applied Mathematics, Weizmann Institute of Science, Rehovot 76100, Israel, ${ }^{2}$ Department of Neurobiology/Brain \\ Research, Weizmann Institute of Science, Rehovot 76100, Israel, and ${ }^{3}$ Human Cortical Physiology and Stroke Neurorehabilitation Section, National \\ Institute of Neurological Disorders and Stroke, National Institutes of Health, Bethesda, Maryland 20892-1428
}

The short-lasting attenuation of brain oscillations is termed event-related desynchronization (ERD). It is frequently found in the alpha and beta bands in humans during generation, observation, and imagery of movement and is considered to reflect cortical motor activity and action-perception coupling. The shared information driving ERD in all these motor-related behaviors is unknown. We investigated whether particular laws governing production and perception of curved movement may account for the attenuation of alpha and beta rhythms. Human movement appears to be governed by relatively few kinematic laws of motion. One dominant law in biological motion kinematics is the $2 / 3$ power law (PL), which imposes a strong dependency of movement speed on curvature and is prominent in actionperception coupling. Here we directly examined whether the 2/3 PL elicits ERD during motion observation by characterizing the spatiotemporal signature of ERD. ERDs were measured while human subjects observed a cloud of dots moving along elliptical trajectories either complying with or violating the $2 / 3 \mathrm{PL}$. We found that ERD within both frequency bands was consistently stronger, arose faster, and was more widespread while observing motion obeying the $2 / 3 \mathrm{PL}$. An activity pattern showing clear $2 / 3$ PL preference and lying within the alpha band was observed exclusively above central motor areas, whereas $2 / 3$ PL preference in the beta band was observed in additional prefrontal-central cortical sites. Our findings reveal that compliance with the $2 / 3 \mathrm{PL}$ is sufficient to elicit a selective ERD response in the human brain.

Key words: 2/3 power law; action-perception coupling; biological motion; event-related desynchronization; motion perception; movement kinematics

\section{Introduction}

Localized power attenuation in an ongoing EEG rhythm, known as event-related desynchronization (ERD), is associated with increased excitability of cortical neurons during sensory, cognitive, and motor processing (Gastaut, 1952; Neuper and Pfurtscheller, 2001a). ERD is linked with motor-related brain functions, mainly execution and imagery of motor actions (Pfurtscheller and Lopes da Silva, 1999; Pfurtscheller et al., 2006). In humans, sensorimotor ERD within alpha and beta frequency bands $(8-13 \mathrm{~Hz}$, known as mu rhythm, and 15-25 Hz, respectively) may reflect cortical activity analogous

Received Dec. 23, 2013; revised Nov. 24, 2014; accepted Dec. 3, 2014.

Author contributions: Y.M., H.H., E.D., A.A., and T.F. designed research; Y.M. and H.H. performed research;Y.M. and H.H. analyzed data; Y.M., H.H., E.D., A.A., and T.F. wrote the paper.

This work was supported by the European Union projects FP7-ICT-248311 AMARSi, EC-ICT- 257695 Vere, and EC FP7-ICT-249858 TANG0, Planning and Budgeting Committee I-CORE Program, and Israel Science Foundation Grant 51/11. H.H. is supported by the Azrieli Foundation. T.F. is an incumbent of the Dr. Moross Professorial chair. We thank Prof. Dov Sagi for supporting this work; Dr. Simon Israeli-Korn, Dr. Shlomit Ben Ami, Irit Sella, and Prof. Martin Giese, Prof. Daniel Bennequin, Prof. Alain Berthoz, and Prof. Moshe Abeles for useful discussions; and Dr. Jenny Kien for editorial help.

The authors declare no competing financial interests.

*Y.M. and H.H. contributed equally to this work.

Correspondence should be addressed to Dr. Yaron Meirovitch, Department of Computer Science and Applied Mathematics, Weizmann Institute of Science, Rehovot 76100, Israel. E-mail: yaron.mr@gmail.com.

DOI:10.1523/JNEUROSCI.5371-13.2015

Copyright $\odot 2015$ the authors $\quad 0270-6474 / 15 / 351627-11 \$ 15.00 / 0$ to the monkey's mirror neuron system (e.g., Hari et al., 1998; Oberman et al., 2005; Perry and Bentin, 2009) because this activity arises during both observation of actions and their execution.

ERD has been extensively used for studying the mechanisms of vision and sensorimotor control (Jasper and Penfield, 1949; Pfurtscheller, 2001) and is of rapidly growing clinical interest (Leocani and Comi, 2006). However, it is unknown whether there are common underlying physical attributes of movement that trigger ERD in both action and perception. Nor has a spatiotemporal ERD pattern in response to motion stimuli been fully characterized.

One potential source of action-perception coupling is the invariant regularities constraining the kinematics of human movement. Because of the considerable redundancy in movement control, any action can be hypothetically performed in many different ways by following many different kinematic profiles (Bernstein, 1967). However, various behavioral and modeling studies have indicated that the CNS generates movements showing specific kinematic regularities (Soechting and Lacquaniti, 1981; Bizzi et al., 2000; Flash and Hochner, 2005). Hence, human movements obey specific kinematic rules, the so-called kinematic laws of motion (e.g., Fitts, 1954; Abend et al., 1982; Flash and Hogan, 1985; Todorov and Jordan, 1998). One such dominant law is the two-thirds power law (2/3 PL) (Lacquaniti et al., 1983), 

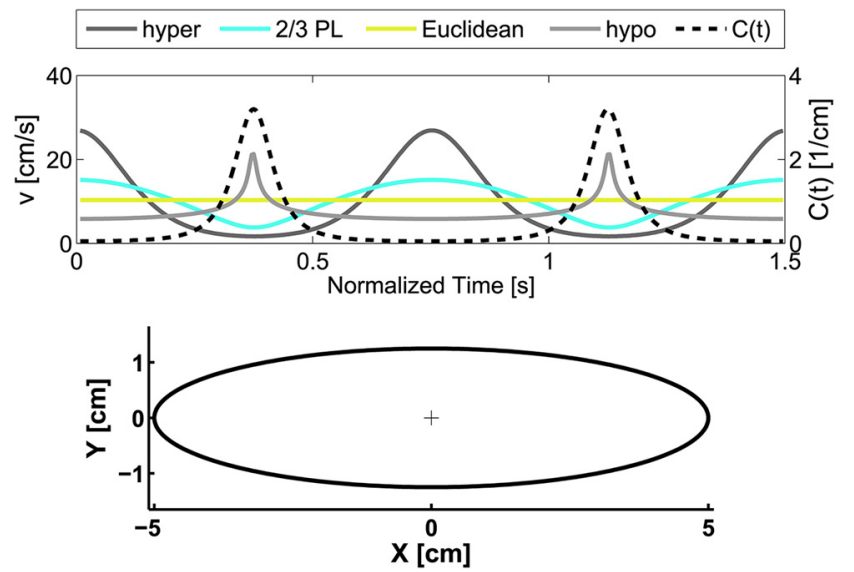

Figure 1. Top, Velocity profiles and instantaneous curvature (C) for the four motion types used as stimuli. Bottom, An illustration of the corresponding elliptical trajectory (an elliptical trace was not shown in the actual presentation).

which was shown to constrain movement generation (Viviani and Flash, 1995; de'Sperati and Viviani, 1997). This law dictates a specific relationship between a movement's temporal and geometric features according to the mathematical expression as follows:

$$
A=K C^{2 / 3} \text {, }
$$

where $A$ is the instantaneous angular velocity of the movement, $C$ is the instantaneous curvature, and the parameter $K$ is a piecewise constant coefficient called the velocity gain factor. Here, instead of using the above formulation, we use an alternative, mathematically equivalent formulation of the 2/3 PL:

$$
V=K R^{1 / 3} \text {, }
$$

where $V$ is the magnitude of tangential velocity and $R$ is the radius of curvature raised to the power $1 / 3$. Although this exponent was repeatedly found to have the value of $1 / 3$ for elliptical trajectories (Lacquaniti et al., 1983), it has other values for other movement paths (Richardson and Flash, 2002). Similarly to the ERD observation, the 2/3 PL constraint was demonstrated both in movement generation and motion perception (Viviani and Stucchi, 1992; Dayan et al., 2007; Casile et al., 2010).

Here we examined the role of this kinematic regularity $(2 / 3$ PL) in eliciting alpha and beta ERD in humans during observation of motion. We compared the strength, spatial distribution, and onset of the ERD elicited while observing this and other motion types.

\section{Materials and Methods}

\section{Participants}

Thirteen paid healthy subjects participated in the experiment $(8$ male, 5 female; age $27 \pm 2.5$ years) after signing a written informed consent form approved by the Weizmann Institute of Science Ethical Committee in accordance with the Helsinki application approved by the Tel-Aviv Sourasky Medical Center. All had normal or corrected-to-normal vision and were right-handed.

\section{Stimuli}

The stimuli consisted of clouds of white dots (Dayan et al., 2007) rigidly moving along elliptical paths against a dark background (Fig. 1). The eccentricity of the elliptical path, $\epsilon$, was fixed at 0.968 (Levit-Binnun et al., 2006) based on the following formula:

$$
\epsilon^{2}=1-\left(\frac{B_{\text {minor }}}{B_{\text {major }}}\right)^{2}
$$

where $B_{\text {major }}$ and $B_{\text {minor }}$ indicate the ellipses' major and minor semi-axes, respectively. The cloud of dots completed one cycle of elliptical trajectory in $1.5 \mathrm{~s}$, corresponding to an average velocity of $9.4 \mathrm{~cm} / \mathrm{s}$. During the EEG recordings, subjects viewed a $9 \mathrm{~s}$ video presenting 6 cycles of the moving cloud of dots. The stimuli moved along the elliptical trajectories with velocity profiles according to four different laws of motion kinematics obeying the following general equation:

$$
V=K R^{\beta} .
$$

The four different conditions were as follows: (1) the $2 / 3$ PL where $\beta=$ $1 / 3$ (2/3 PL; Fig. 1, cyan); (2) Euclidean where $\beta=0$; the speed remained constant for the entire geometric path (Fig. 1, yellow); (3) hypo where $\beta=-1 / 3$; the speed increased during the curved segments of the path and decreased during the straighter segments (representing an inverse $2 / 3$ PL motion; Fig. 1 , bright gray); and (4) hyper where $\beta=2 / 3$; the movement was slower during more curved segments of the ellipse and faster during the straighter segments, relative to the 2/3 PL (Fig. 1, dark gray). Stimulus delivery and response acquisition were controlled by a PC using the Presentation software (Neurobehavioral Systems). Stimuli were presented on a 19 inch Mitsubishi Diamond Pro 930SB color monitor with a refresh rate of $100 \mathrm{~Hz}$ in an otherwise dark environment.

In motion perception, the periodic movement of a dot along an elliptical trajectory is perceived as most uniform and smooth for velocity profiles obeying the 2/3 PL (Viviani and Stucchi, 1992). This phenomenon has been extensively studied in motor control, especially given the theoretically demonstrated unique relation between the 2/3 PL and smoothness maximization while following different figural forms. Particularly, for elliptical trajectories, smoothness maximization was shown to be consistent with the $2 / 3$ PL with some dependence of the exponent on the ellipse's eccentricity. Although, for other more complex shapes, other exponents resulted in maximally smooth movements (Richardson and Flash, 2002), the elliptical trajectories used in this study were carefully chosen to elicit modulations in the smoothness of the motion kinematics that is maximized by the $2 / 3 \mathrm{PL}$.

\section{Experimental procedure}

Each trial began with a fixation period. To reduce the predictability of stimulus onset, the duration of the fixation period was set at $6 \mathrm{~s} \pm \Delta_{t}$ where $\Delta_{t}$ was randomly selected from the set $\left\{-1,-\frac{1}{2}, 0, \frac{1}{2}, 1\right\}$. At stimulus onset, a $9 \mathrm{~s}$ video presenting the moving cloud of dots was initiated. The video also continuously displayed a white fixation cross at the center of the elliptical path. During the last $0.5 \mathrm{~s}$ of the video presentation, the fixation cross randomly changed color to either green or red. To monitor eye fixation and general alertness, the subjects were instructed to report the color of the fixation cross at the end of each trial by pressing a corresponding keyboard key button. A session was composed of 4 blocks of 20 trials each, the blocks being separated by a rest period of 2-4 min. Each block displayed all four motion types in a randomized order ( 5 trials for each motion type).

\section{EEG recording and data analysis}

EEG signals were continuously recorded at 64 cortical sites using Ag$\mathrm{AgCl}$ pin-type active electrodes mounted on an elastic cap according to the extended 10-20 system (ActiveTwo, BioSemi, impedance $<5 \mathrm{k} \Omega$ ). Two additional reference electrodes were placed at the right and left mastoids. Recordings were filtered online using the ADC's decimation low-pass filter with a $3 \mathrm{~dB}$ attenuation point at one-fifth of the sampling rate $(256 \mathrm{~Hz})$. EEG data were rereferenced offline to the average of the reference mastoid electrodes. Recordings were synchronized with the onset of the visual stimulus.

Data from 15 subjects were recorded. All trials were visually inspected for movement artifacts, and highly noisy trials ( $<10 \%$ of all trials) were discarded. Data consisting of low alpha band activity ( 1 subject) were discarded because of low signal-to-noise ratio. The data of a further subject were discarded because of the lack of any power attenuation in 
A

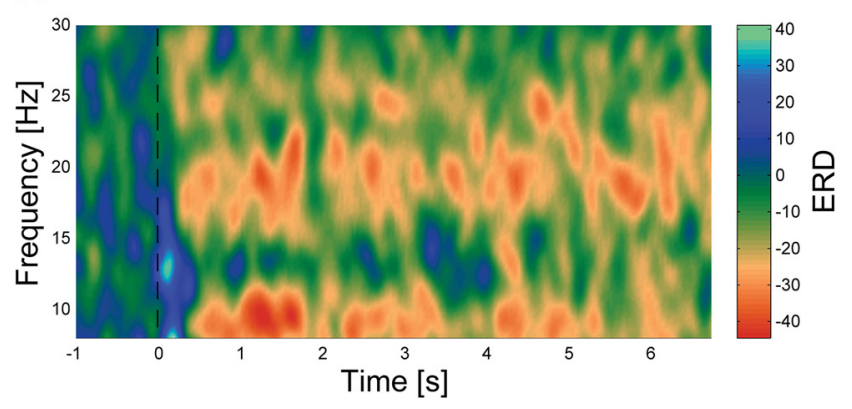

B
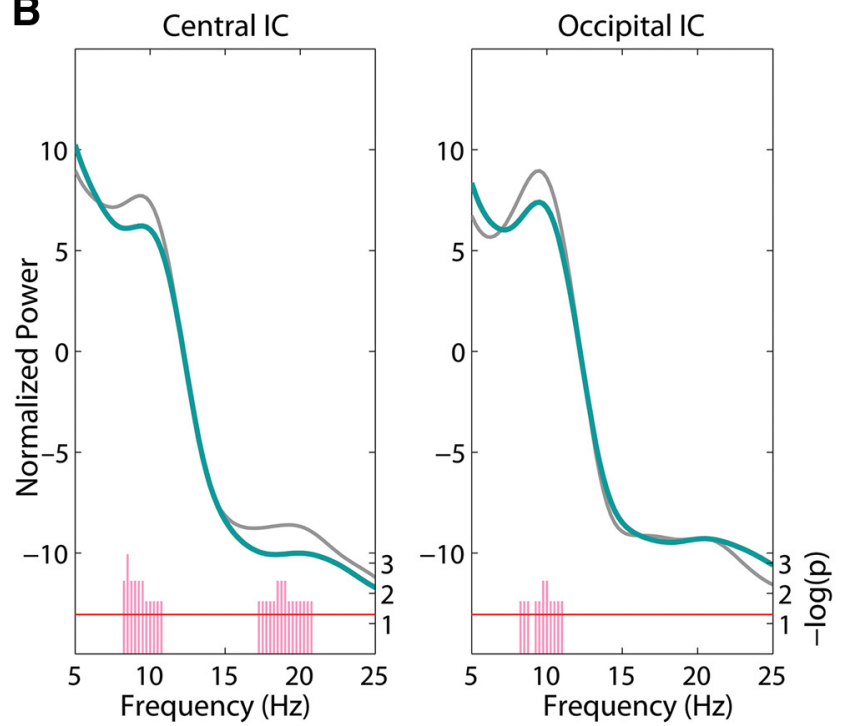

Baseline

Stimulus

Figure 2. A, Time-frequency analysis of the power change in the spectrum of EEG signals in electrode $\mathrm{Cz}$ averaged across all trials of all subjects for the $2 / 3 \mathrm{PL}$ motion. Dashed line indicates stimulus onset. Red represents larger ERD. ERDs were found both in alpha and in beta frequency bands. $\boldsymbol{B}$, The normalized power across two time frames (baseline, stimulus) of two ICs: central component (left) and occipital component (right; see EEG recording and data analysis). The normalized power was calculated for all frequencies and subjects and collapsed across the four experimental conditions. Right, $y$-axis represents the resulting $p$ values from sign tests examining the hypothesis that baseline power is larger than stimulus power. Red line indicates $p=$ 0.05 .

response to all four motion types (e.g., Nikouline et al., 2000; Lepage and Théoret, 2006).

The data from the remaining 13 subjects consisted of 20 trials per motion type, with four different motion types. The EEG data were lowpass and high-pass filtered offline with $40 \mathrm{~Hz}$ and $2 \mathrm{~Hz}$ cutoff frequencies, respectively (by applying FIR and IIR filters using EEGLAB MATLAB toolbox) (Delorme and Makeig, 2004). Independent component analysis was applied to each of the subjects' EEG datasets, and components related to eye blinks and eye movements were discarded based on a standard approach using the ADJUST plugin (Mognon et al., 2011). EEG power spectra were obtained by computing discrete Fourier transforms using a fixedlength sliding window of 128 samples ( $0.5 \mathrm{~s}$ epochs) and averaged by a Hamming window allowing for a frequency resolution of $2 \mathrm{~Hz}$ with window overlap of 124 samples.

Based on previous studies of movement (Crone et al., 1998; Pfurtscheller and Lopes da Silva, 1999; Neuper and Pfurtscheller, 2001a) and clinical studies involving motor imagery (e.g., Kühn et al., 2006), the EEG data were separated into two frequency bands for further analysis. The alpha band range was adapted for each subject because it has been reported that the specific band within the alpha range that is sensitive to action varies among subjects (e.g., Muthukumaraswamy and Johnson,
2004). The beta band range was analyzed within $19-21 \mathrm{~Hz}$ (Pfurtscheller et al., 1997; Neuper and Pfurtscheller, 2001b; Hatsopoulos, 2009). To determine the alpha band range corresponding to motor processing in each subject, the power modulations in the motor-related central electrode $(\mathrm{Cz})$ were averaged across all motion types and time, from $1 \mathrm{~s}$ after stimulus onset until the end of the recording. For each subject, we identified a frequency range between 8 and $13 \mathrm{~Hz}$ at which the largest power attenuation occurred. A $2 \mathrm{~Hz}$ window around this frequency $(1 \mathrm{~Hz}$ below and $1 \mathrm{~Hz}$ above) was used for further analysis.

ERD signals were calculated as follows: for each subject and electrode, we obtained the mean power spectra across trials from the same motion type (for a display of spectrogram of electrode $\mathrm{Cz}$, see Fig. $2 A$ ). For each frequency, ERD signals were defined as the percentage change in power spectrum using a typical ERD time window of $0.5-1.5 \mathrm{~s}$ after stimulus onset relative to the average power during a reference period of 3.5-0.5 s before the stimulus onset. A more negative ERD indicated stronger power attenuation relative to the reference period and was considered to reflect increased motor cortex excitability. The ERD signals were downsampled to a rate of 8 samples per movement cycle $(1.5 \mathrm{~s})$ both for the analyses of the ROI (Fig. 3) and of the extended ROI (see below). ERD signals were normalized by log transformation before the parametric statistical tests. For the spatiotemporal analysis of the ERD signals, they were down-sampled to a rate of $64 \mathrm{~Hz}$ to reduce between-sample dependencies.

Independent component analysis was conducted to validate our model-driven analysis and examine the spectral properties of the central alpha and beta ERDs, within and across subjects. To define the electrode weights of the different independent components (ICs), epoched data within a time window of -1.5 to $1.5 \mathrm{~s}$, around stimulus onset, were used (Bell and Sejnowski, 1995; Makeig et al., 2004). The component maximizing the contribution from the vertex electrode was determined for each subject independently (central IC). The power spectra defined by these components were calculated within two time frames (encompassing the total length of each trial): one corresponding to the baseline (baseline, $4 \mathrm{~s}$ before stimulus onset) and the second corresponding to the stimulus presentation (stimulus, $9 \mathrm{~s}$ following onset), separately.

The central IC was used to investigate desynchronization, or power reduction, as a function of frequency band. For comparison, we investigated an occipital IC (the independent component governed by the $\mathrm{Oz}$ electrode) as well. Figure $2 B$ shows the normalized power of the central (left panel) and occipital (right panel) ICs, separately calculated for two time frames (baseline and stimulus, see above). The normalized power was calculated for all frequencies and subjects and collapsed across the four experimental conditions. A corresponding sign test was performed for testing the hypothesis that the "baseline" power is larger than the "stimulus" power. The resulting $p$ values are shown in the pink bars (the $y$-axis on the right). The red line indicates $p=0.05$. This analysis shows reduced power around the 10 and $20 \mathrm{~Hz}$ frequency bands (rolandic rhythms). Unlike the central IC power reduction of rolandic rhythms, power reductions in the occipital IC were observed only for the alpha frequency band (associated with a visual response).

\section{Across-subjects analysis}

For each subject, condition, and frequency band, topographic maps of the ERD signals were calculated by averaging these signals. To construct the ERD across-subjects significance maps, the ERD amplitudes obtained from all the electrodes were interpolated over a scalp model represented in the Talairach coordinate space (Talairach and Tournoux, 1988) using "natural neighbor" interpolation (Sibson and Thomson, 1981) in MATLAB (MathWorks) for visualization purposes. Sign tests for each electrode were then conducted using the ERD amplitudes derived for all subjects $(n=13)$. For each motion type, electrodes with $<5 \%$ change in median ERD across subjects (sign test, $p>0.0008$; Bonferroni correction $n=64)$ were considered nonsignificant electrodes and were labeled "nonrobust electrodes" (Fig. 4A, gray). Electrodes showing significantly low $p$ values across subjects $(p<0.0008)$ were considered to represent robust ERD activities across subjects at that cortical site and were thus designated "robust electrodes" (Fig. $4 A$, color-coded based on the corresponding grand average ERD). The $p$ values of the sign tests were also 
interpolated using the interpolation method described above. A statistical threshold of $p=$ 0.0008 indicated that $>10$ subjects showed a power attenuation response stronger than a $5 \%$ change in power for the respective electrode and motion type. A similar approach for evaluating stability across trials was used by Pfurtscheller and Lopes da Silva (1999).

\section{Spatiotemporal grand average analysis}

The above across-subjects analysis aimed to characterize spatial ERD patterns in response to different motion kinematics. Unlike this analysis that focused on a restricted time window (0.5-1.5 s following stimulus onset) and on estimating the magnitude of the ERD responses to each motion type across subjects (the response robustness), in the next portion of our analysis we investigated the grand average across subjects of the entire $9 \mathrm{~s}$ recording time. This enabled us to assess the temporal persistency of the ERD in each electrode and, subsequently, across clusters of electrodes.

Grand average temporal ERD profiles were computed separately for each electrode, frequency band, and motion type by averaging ERD for each time bin (see below) during the entire 9 s recording time. Stimuli were continuously presented, and the ERD was generally not followed by an event-related synchronization (Fig. 2A, ERD spectrogram). Accurately examining the temporal patterns of the ERD was not possible without a defined model of the underlying distribution of ERD over time and across the experimentally used motion types. However, comparison across the different motion types and electrodes required no $a$ priori parametric assumptions. Our temporal assessment of the ERD profiles was based on the assumption that samples widely separated in time are statistically independent (Halliday and Rosenberg, 1999; Kühn et al., 2006). The motion type generating the largest ERD was determined for each electrode and time bin $(15.62 \mathrm{~ms})$, resulting in one temporal series ("signature") per electrode, which considered all motion types. Figure $5 A$ (top) shows the temporal signature of all electrodes in the alpha band, where each color represents a different motion type using the same color code as in Figure 1: cyan represents $2 / 3 \mathrm{PL}\left(\beta=\frac{1}{3}\right)$; yellow represents

Euclidean $(\beta=0)$; bright gray represents hypo $\left(\beta=-\frac{1}{3}\right)$; dark gray represents hyper $\left(\beta=\frac{2}{3}\right)$. The "barcode" (Fig. $5 A$, bottom) illustrates the temporal signature in the alpha band for electrode C6.

Clustering analysis. To detect shared spatiotemporal patterns signifying coactivation of electrodes, the ERD temporal signature across electrodes was investigated. Hierarchical clustering of the electrodes expressed the similarity of the time series (temporal signatures) among all electrodes (Fig. 5A, top). Such similarity was based on the Hamming distance between the signature functions of all electrodes (using MATLAB function "linkage" with the "complete" distance parameter). The derived clusters are shown in the $y$-axis of the dominance onset figures (Fig. $5 B$ ), where each cluster was designated by a letter (A-E and $\mathrm{F}$ for the alpha band and $\mathrm{A}^{\prime}-\mathrm{E}^{\prime}$ and $\mathrm{F}^{\prime}$ for the beta band). The clusters' spatial distribution is illustrated on the schematic brain map in Figure $5 B$ (top for the alpha band, bottom panel for the beta band).
Dominant motion type. To characterize the temporal signature of each electrode, the motion type eliciting the persistently strongest ERD over time was termed the "dominant motion type." The persistency was defined in statistical terms. It related to a time period starting at the "dominance onset" time and continuing for all the subsequent time bins until the end of the $9 \mathrm{~s}$ recording period. The dominant motion type was determined by a binomial test. The null hypothesis assigned each motion type a chance level probability of being dominant within any specific time bin $\left(p=\frac{1}{4}\right)$. For each motion type (under its null hypothesis) and time bin, we computed the probability of obtaining the examined temporal signature.

Because we were interested in responses with early onsets, a motion type was considered "dominant" based on three criteria: (1) its corresponding probabilities based on the binomial test detailed above became persistently lower than those of the other motion types from a given point in time onwards. This was termed dominance onset. (2) Dominance onset was detected within the first $5 \mathrm{~s}$ after stimulus onset; and (3) its corresponding probabilities (see the binomial test described above) were 
A

Alpha

Beta
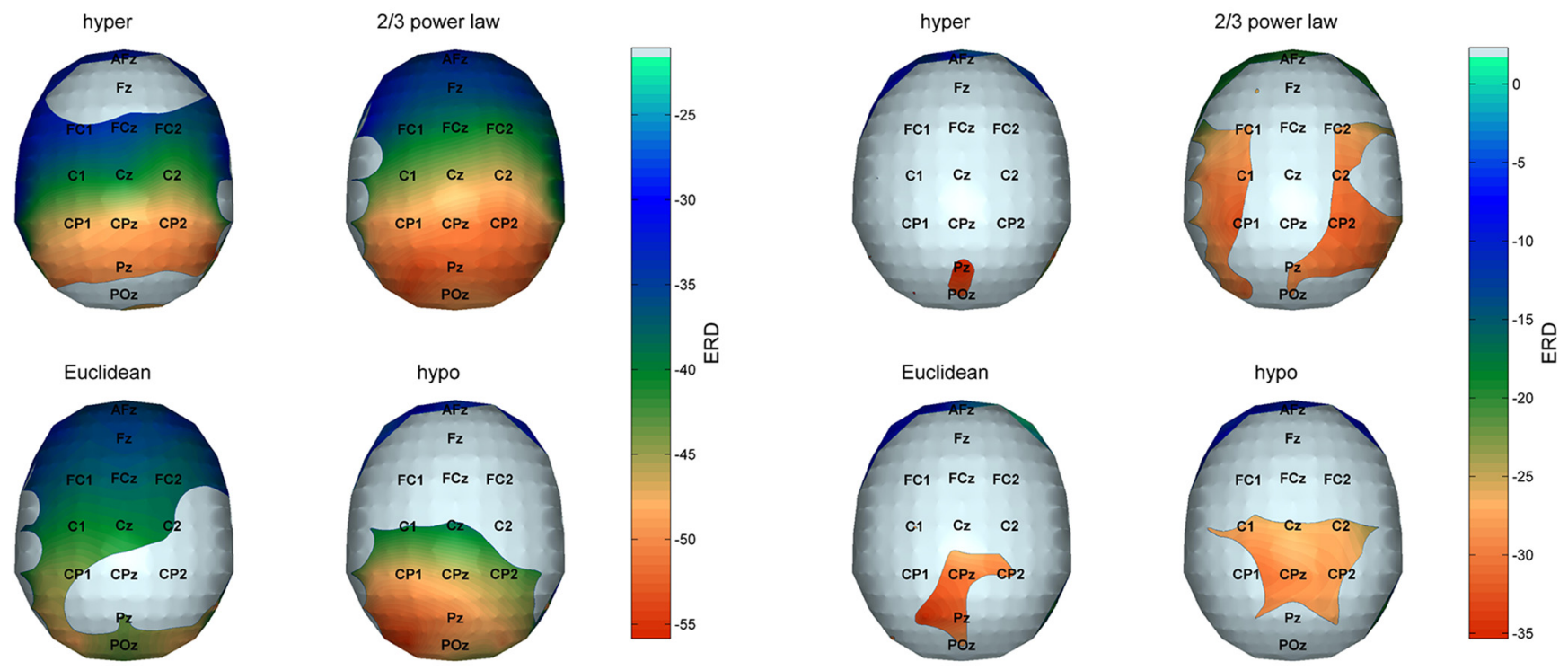

B

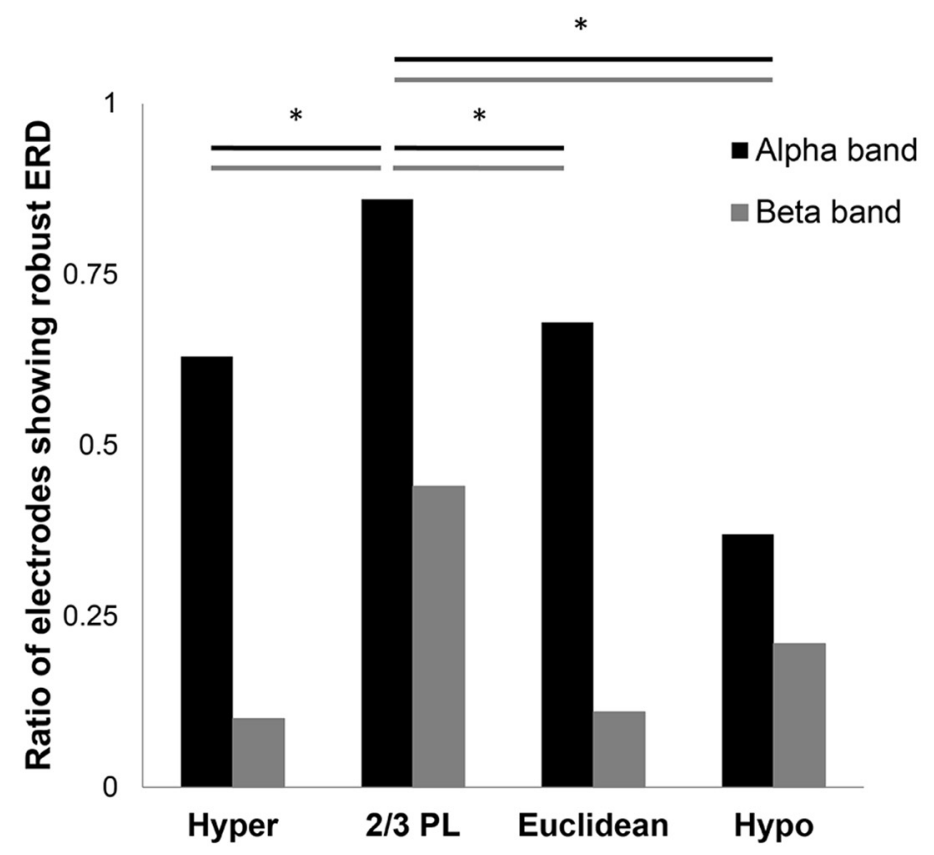

Figure 4. $\quad \boldsymbol{A}$, Across-subjects analysis of alpha ERD (left) and beta ERD (right) depicted for each motion type. The consistency of response across subjects at each electrode is marked by the contour corresponding to $p=0.0008$ of the $p$ values superimposed on the scalp. Gray represents nonrobust areas (sign test, Bonferroni corrected, $n=64$ ). ERD averages are color-coded: red represents a larger response; blue represents more moderate responses. $\boldsymbol{B}$, Bars represent the proportions of electrodes showing robust ERD, sorted by frequency band and motion type. ${ }^{*} p<0.05$.

lower than a predefined threshold $\left(p=10^{-8}\right)$. This conservative threshold was selected based on a separate analysis of the entire activity, including baseline, to avoid a dominance onset that appeared before the stimulus onset. Therefore, there were cases where the strongest ERD could have been attributed to different motion types during different time bins, based on the temporal signature analysis but, from the point of view of statistical analysis, a single motion type acquired sufficient statistical tendency to be considered dominant. Figure $5 A$ (right panel) exemplifies such a case by illustrating the power of this statistical tendency corresponding to the temporal signature of the C6 electrode (Fig. 5A, bottom left). For each motion type, we counted the accumulated number of time bins during which that motion type elicited the strongest ERD. The statistical power here was defined by $-\log (\mathrm{P})$ where $\mathrm{P}$ corresponded to the probability (for each motion type) of obtaining the observed count, which was updated for each time bin. Higher $-\log (\mathrm{P})$ values represent lower probabilities for accepting the null hypothesis based on the binomial test. This probability was calculated for each time point based on all prior time bins. Thus, although this temporal signature analysis showed fluctuations with respect to the motion type eliciting the strongest ERD, in probabilistic terms the $2 / 3 \mathrm{PL}$ was persistently pre- 
A
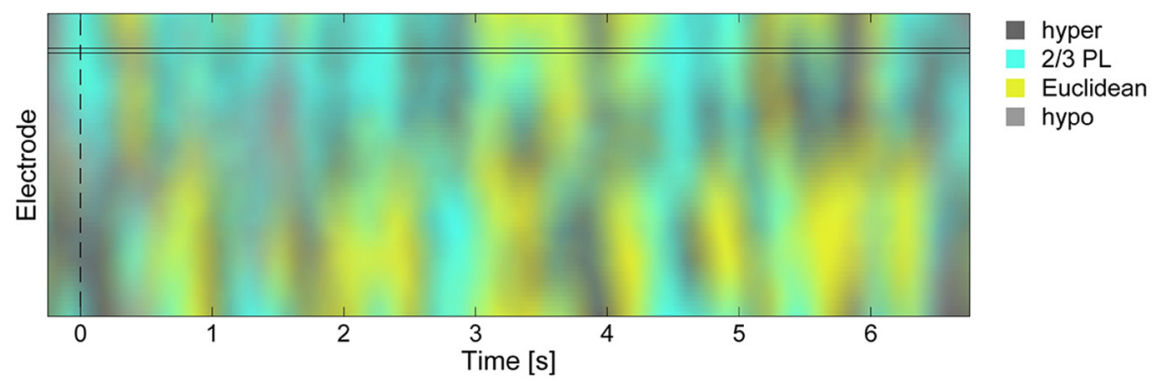

C6

口十⿴囗十口

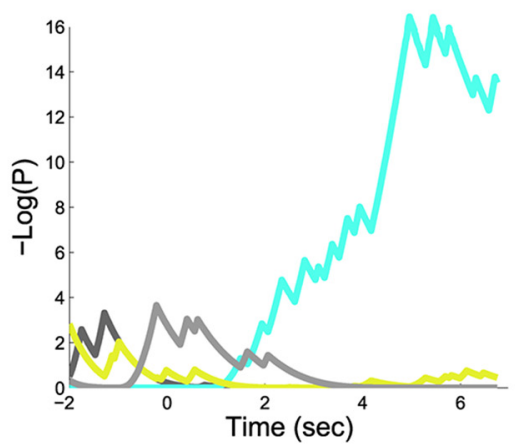

B

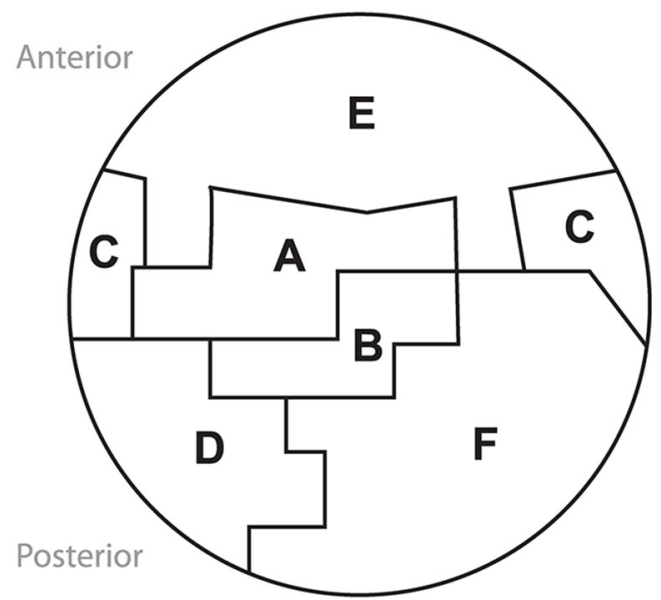

\section{Electrodes}

a Dominance Onset
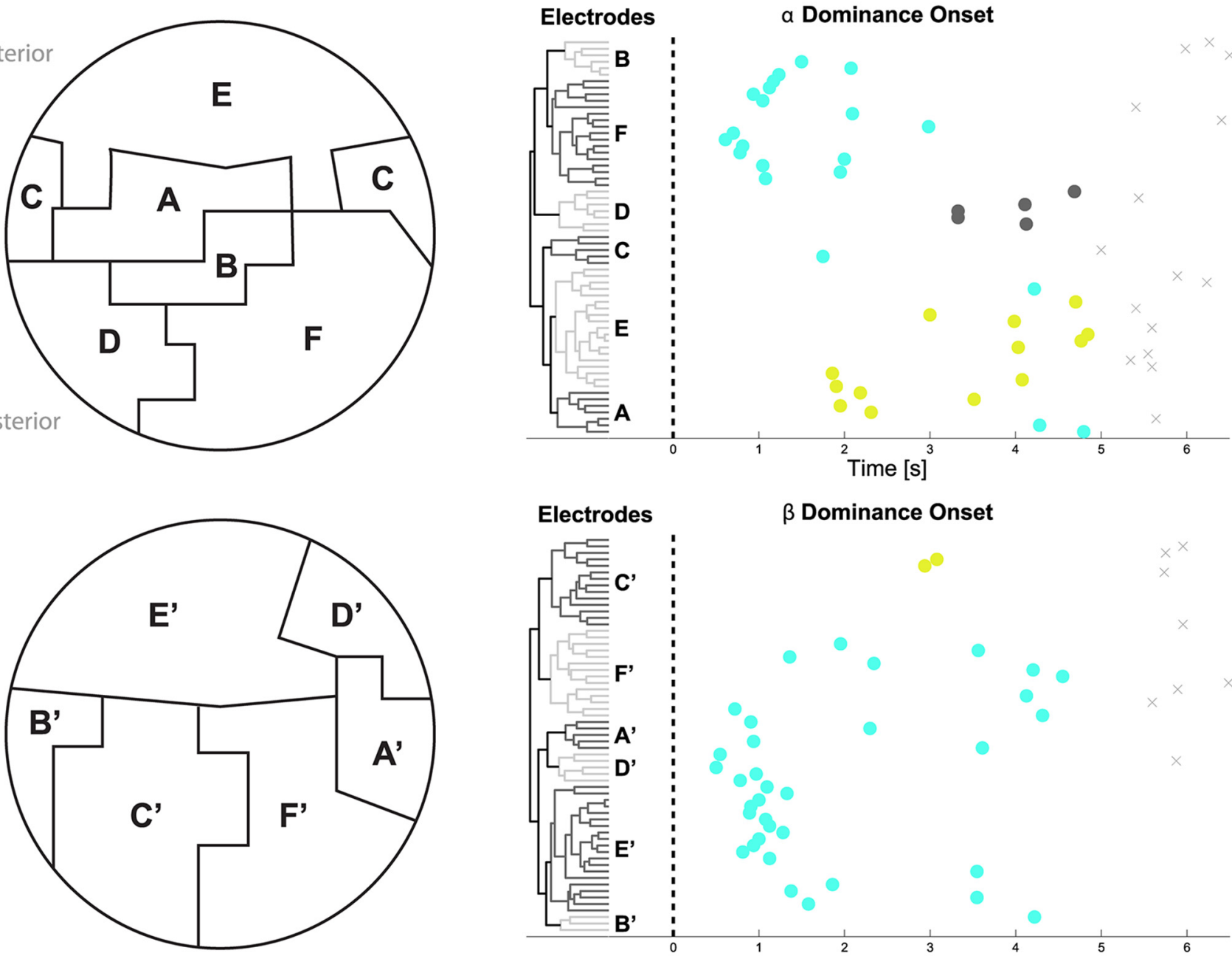

Electrodes

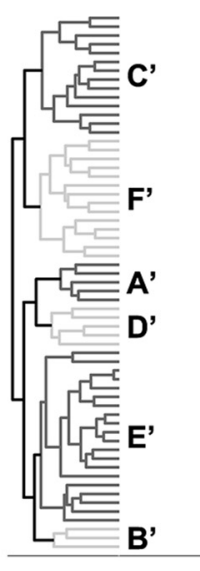

$\beta$ Dominance Onset

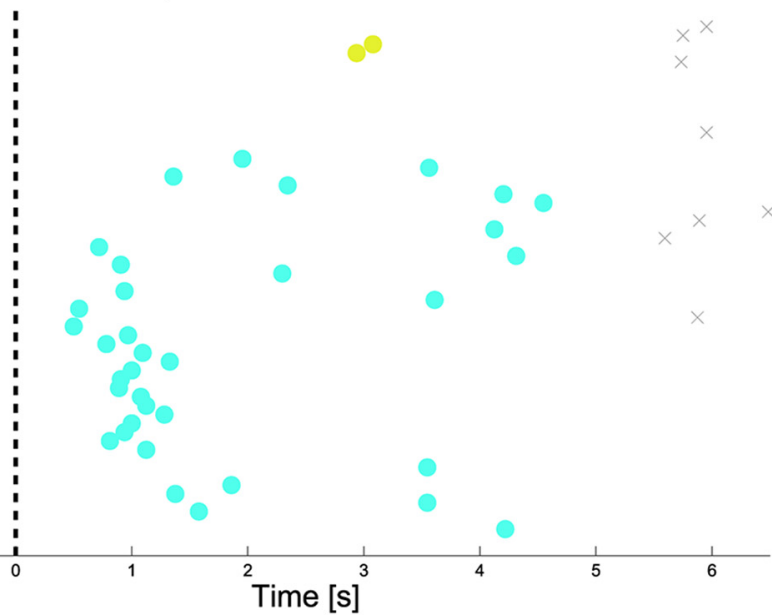

Figure 5. A, Upper left, Temporal signature of all recorded electrodes over time (alpha band). The temporal signatures were smoothed across time and electrodes to allow visualization. Dashed black line indicates stimulus onset. Bottom left, Temporal signature of the 66 electrode. This time series shows which motion type generated the largest ERD for each time bin. Colors indicate motion types: cyan represents $2 / 3$ PL; yellow represents Euclidean; bright gray represents hypo; dark gray represents hyper. Right, The power of the statistical tendency corresponding to the temporal signature of the 6 electrode. The statistical power here was defined by $-\log (\mathrm{P})$ where $\mathrm{P}$ corresponds to the probability (for each motion type) of obtaining the observed temporal dominance under the null hypothesis. Higher $-\log (\mathrm{P})$ values represent increased dominance. Thus, although this temporal signature shows fluctuations of the motion type eliciting the strongest ERD, in probabilistic terms the 2/3 PL was persistently preferred. B, Spatiotemporal analysis for the alpha (top) and beta bands (bottom). Right, Ordinate represents the electrodes arranged in the clusters while minimizing the distance between adjacent electrodes in the hierarchical tree (MATLAB function dendrogram). Also shown are their corresponding dominant motion types and dominance onset. The dominant motion type generated a persistently larger ERD than other motion types. The dominance onset was the earliest time bin from which a given motion type elicited a persistently larger ERD than all other motion types. " $X$ " markers indicate electrodes for which a motion type was dominant at least $5 \mathrm{~s}$ following onset. The letters of the clusters match the letters in the schematic brain maps on the left. Colors as in $\boldsymbol{A}$. 
ferred (i.e., was the most dominant motion type). This type of analysis was therefore used to determine the dominant motion type and to compare dominance onsets for different electrodes.

\section{Results \\ Motor ROI}

We first examined whether a stronger sensorimotor ERD was more evident in response to observing the $2 / 3 \mathrm{PL}$ motion type than when observing the other types of motion. The $\mathrm{Cz}, \mathrm{FCz}$, and $\mathrm{CPz}$ electrodes, covering part of the medial sensorimotor cortex, comprised the motor ROI, whereas the $\mathrm{Oz}, \mathrm{POz}$, and $\mathrm{Pz}$ electrodes, covering part of the parieto-occipital cortex, were chosen to represent the nonmotor control ROI. For this analysis, a time window of $0.5-1.5 \mathrm{~s}$ relative to the stimulus onset was chosen, as is common in ERD research for the alpha and beta band ranges (Pfurtscheller and Lopes da Silva, 1999). ERD temporal profiles were averaged across trials for each subject, motor ROI electrode, and condition. Next, to compare each of the other motion types with the $2 / 3 \mathrm{PL}$ motion, ERD profiles were analyzed by a repeated-measures ANOVA, followed by Dunnett's correction for multiple comparison. ERD in the alpha band was significantly modulated by motion type in the $\mathrm{Cz}$ electrode $\left(F_{(3,260)}=4.75\right.$, $p=0.003$ ), where the $2 / 3 \mathrm{PL}$ motion generated the largest ERD $(p<0.05)$. ERD in the beta band was significantly modulated by motion type in the FCz electrode $\left(F_{(3,260)}=2.69, p=0.047\right)$, where the $2 / 3$ PL motion generated the largest ERD $(p<0.05)$. In both frequency bands, none of the motor ROI electrodes showed significant preference for any of the other motion types.

Further examination revealed not only that the $2 / 3$ PL motion type resulted in the strongest response, but also that the larger the divergence from this kinematic regularity, assessed according to the $\beta$ value for each motion type, the weaker was the response. Figure 3 shows the temporal distribution (ERD across time, sampled at $64 \mathrm{~Hz}$ ) of the grand average ERD for the motor and the nonmotor control ROIs (right and left panels, respectively) and for the alpha and beta bands (upper and bottom panels, respectively). Figure 3 displays average ERD across the ROI electrodes, whereas the statistical analysis discussed above was applied to each electrode individually. Increased motor cortex excitability is reflected in a larger decrease in power relative to the baseline (i.e., in an ERD with larger magnitude). In the alpha band, 2/3 PL motion elicited stronger ERD in both the motor and nonmotor ROIs. In the motor ROI, ERD was modulated by the motion kinematics, decreasing gradually and monotonically as the motion type diverged from the $2 / 3 \mathrm{PL}$. In the beta band, the $2 / 3 \mathrm{PL}$ motion elicited stronger ERD in response to the $2 / 3 \mathrm{PL}$ in the motor ROI but not in the control ROI.

\section{Extended ROI}

To determine whether other brain areas (not necessarily motor) were modulated by kinematic regularities, we performed an analysis as described above on all 64 electrodes, using $\alpha=0.0008$ (based on Bonferroni correction taking into account the number of electrodes, $n=64$ ) to account for multiple analyses. This showed that no further electrodes were modulated by motion type in the alpha band (data not shown); thus, alpha activity responding early to $2 / 3 \mathrm{PL}$ motion was confined exclusively to central motor and premotor sites. An increased beta band ERD in response to $2 / 3 \mathrm{PL}$ motion was detected in several additional frontal and right prefrontal electrodes (data not shown): Fpz, $\mathrm{AF} 4, \mathrm{~F} 6, \mathrm{FC} 4$, and $\mathrm{AFz}\left(F_{(3,240)}=15.65, p<0.0001 ; F_{(3,260)}=\right.$ $11.57, p<0.0001 ; F_{(3,260)}=10.22, p<0.0001 ; F_{(3,260)}=8.63, p<$ $\left.0.0001 ; F_{(3,260)}=10.59, p<0.0001\right)$. Importantly, no electrode showed significant preference for any of the other three motion types in either the alpha or the beta bands.

\section{Across-subjects analysis}

We quantified the "robustness" (across-subjects significance) of the spatial ERD patterns (topographies) independently for each motion type. Such topographic analysis may reveal the spatial extent of ERD that appears consistently across subjects for each of the frequency bands, an observation that has potential functional implications.

Figure $4 A$ shows the across-subjects significance map of ERD in the alpha and beta bands for each of the four motion types (left and right panels, respectively). Colored in gray are electrodes that resulted in no across-subjects significance. The electrodes in which there was across-subjects significance $(p<0.0008)$ were color-coded based on the grand average ERD. For both frequency bands, the response to the $2 / 3 \mathrm{PL}$ was more widespread than to any other motion type.

\section{Alpha activity}

The alpha band average response was strong at the central motor areas (colored sites, $\mathrm{Cz}, \mathrm{CPz}$ ) for all motion types. The motion type eliciting the most spatially extensive response was $2 / 3 \mathrm{PL}$ motion (Fig. 4A, left). Responses to the hypo motion type, kinematically the most divergent from the 2/3 PL motion, showed the least robust alpha ERD (gray area), suggesting a steadily decreasing response as the motion type became less regularized by the $2 / 3$ PL (i.e., less "biological"; fitting with Fig. 3).

\section{Beta activity}

The beta band average response to the $2 / 3 \mathrm{PL}$ motion revealed bilateral activity in premotor and motor sites (Fig. $4 A$, right, colored sites). The hyper and Euclidean motion kinematics revealed a local robust activity in the sensorimotor and parieto-occipital sites in contrast to the majority of gray electrodes (nonsignificant across subjects). The hypo motion kinematics generated a broader robust ERD response at motor sites. This result is consistent with other studies, such as Dayan et al. (2007), who also found strong neural activity in specific brain areas in response to unnatural motion types, although this response was weaker and considerably less extensive than the response to the 2/3 PL (Gangitano et al., 2004; see also Costantini et al., 2005). Furthermore, a recent study (Pavlidou et al., 2014) found a prominent effect of the biomechanical plausibility of an observed action on the beta band activity, with stronger attenuation of beta power in response to implausible than to plausible motion patterns in the left temporal parieto-occipital and sensorimotor brain areas.

The spatial extent of the response was quantified by the number of electrodes showing robust ERD, regardless of its magnitude or the electrode identity (see also Spatiotemporal grand average analysis). Figure $4 B$ displays the proportion of electrodes showing a robust ERD response for each motion type and frequency band. To estimate the differences between the 2/3 PL proportion and the proportion of each of the other motion types within each frequency band, we applied a $\chi^{2}$ test. The $2 / 3 \mathrm{PL}$ motion type showed the most spatially extensive robust activity of all four motion types (evident in the larger proportions) in both alpha (black bars, $2 / 3$ PL vs hyper; $\chi^{2}=7.8 ; p=0.005,2 / 3$ PL vs Euclidean; $\chi^{2}=5.1 ; p=0.02,2 / 3$ PL vs hypo; $\chi^{2}=31.3$; $p<0.001, \mathrm{df}=1$ for each comparison) and beta (gray bars, $2 / 3$ PL vs hyper; $\chi^{2}=19.3 ; p<0.001,2 / 3$ PL vs Euclidean; $\chi^{2}=17.3$; $p<0.001,2 / 3$ PL vs hypo; $\chi^{2}=8.0 ; p=0.004, \mathrm{df}=1$ for each comparison) frequency bands. Moreover, across frequency 
bands, the robust alpha activity was more widespread in this time interval than the robust beta activity (higher proportion for all motion types, hyper; $\chi^{2}=39.2, p<0.001,2 / 3$ PL; $\chi^{2}=22.9, p<$ 0.001 , Euclidean; $\chi^{2}=42.5, p<0.001$, hypo; $\chi^{2}=3.8, p=0.05$ ).

\section{Spatiotemporal grand average analysis}

Figure 5 compares ERD across all four motion types for each electrode and at each time bin. The figure is based on the temporal signature of the individual electrodes, as well as on the coactivation of different electrodes. First, we computed the temporal signature for each electrode (the temporal signature of electrode C6 is presented in Fig. 5A, bottom, for illustration purposes only). ERD signals were averaged across trials and subjects. Then, for each electrode, a temporal signature was constructed by finding the motion type that elicited the largest ERD at any given time bin. Colors indicate motion types as in Figure 1: cyan represents 2/3 PL $\left(\beta=\frac{1}{3}\right)$; yellow represents Euclidean $(\beta=0)$; bright gray represents hypo $\left(\beta=-\frac{1}{3}\right)$; dark gray represents hyper $\left(\beta=\frac{2}{3}\right)$. To construct a spatiotemporal map, clustering analysis was applied (see Spatiotemporal grand average analysis). Electrodes were clustered based on the similarity of their temporal ERD signature (for the temporal signatures of all electrodes, see Fig. 5A, top). Second, we examined this temporal signature for each electrode to determine whether the response to any motion type was persistently larger (dominant) than to all other motion types, considering the full $9 \mathrm{~s}$ recording time (see Spatiotemporal grand average analysis). Dominance onset indicates the earliest time bin at which a given motion type became persistently dominant.

Figure $5 B$ (right) shows the following: (1) dominant motion type (indicated by color, as in Fig. 5A), (2) dominance onset (indicated along the $x$-axis), and ( 3 ) the corresponding cluster for each electrode (indicated by a letter next to the $y$-axis). If dominance onset was $>5 \mathrm{~s}$ after stimulus onset, an " $\mathrm{X}$ " marker was used. The clusters are indicated by a dendrogram tree on the left. Electrodes, which were clustered together, are indexed by the same letter (A-E and $F$ for the alpha band and $A^{\prime}-E^{\prime}$ and $F^{\prime}$ for the beta band). Figure $5 B$ (left) shows a schematic brain map with the electrode clusters. Figure $5 B$ shows the results of the analysis for the alpha (top) and beta (bottom) bands. The 2/3 PL (cyan) was the earliest dominant motion type in both frequency bands, becoming dominant within $1 \mathrm{~s}$ after stimulus onset. No other motion type generated such an immediate response. In addition, the $2 / 3 \mathrm{PL}$ was the prevalent dominant motion type in the beta band.

The response to the $2 / 3 \mathrm{PL}$ (cyan) in the alpha band started more posteriorly (cluster F) and in the beta band more anteriorly (clusters $\mathrm{D}^{\prime}, \mathrm{E}^{\prime}$ ). In the alpha band, the most immediately reactive clusters were the central motor and right post-central clusters, which showed a preferred response to $2 / 3 \mathrm{PL}$ motion. Euclidean motion (yellow) became dominant $>2 \mathrm{~s}$ after stimulus onset in more anterior regions (clusters E, A). The hyper motion type (dark gray) exhibited the most delayed dominance onset, $3 \mathrm{~s}$ after stimulus onset, in a restricted left parietal region (cluster D). No electrode showed a preference for the hypo condition (bright gray). Overall, the grand average spatiotemporal analysis of the alpha band response revealed that the $2 / 3$ PL preference showed the most rapid onset of all four motion types. Moreover, in line with previous ROI analyses, 2/3 PL ERD was focused at the sites of the central electrodes.

In the beta band, the majority of electrodes showed a preferred response to the $2 / 3$ PL motion type $\left(\beta=\frac{1}{3}\right.$, cyan $)$; thus, the beta band 2/3 PL ERD was more widespread than the alpha band. The most immediate dominance was that of the $2 / 3 \mathrm{PL}$ starting $1 \mathrm{~s}$ after stimulus onset. This was seen in right precentral and right temporal clusters (clusters $\mathrm{D}^{\prime}, \mathrm{A}^{\prime}$ ) and later in central and frontal regions (clusters $\left.\mathrm{E}^{\prime}, \mathrm{B}^{\prime}, \mathrm{F}^{\prime}\right)$. The Euclidean motion type $(\beta=0$, yellow) was dominant in 2 left parietal electrodes (cluster $C^{\prime}$ ). Finally, the 2/3 PL preference was more widespread for the beta than for the alpha band but was similar across bands in its immediate response onsets.

\section{Discussion}

Here we found the imprints of the kinematics of observed motion on alpha and beta ERD. The ERD in both alpha and beta bands evoked by motion obeying the $2 / 3 \mathrm{PL}$ was stronger than that evoked by all other motion types. Alpha ERD selectivity for $2 / 3$ PL motion was mainly observed at motor areas, whereas beta ERD responses selective to motion obeying this law were recorded at motor and prefrontal nonmotor sites. ERD research usually considers only early responses. Here, our spatiotemporal analysis revealed that $2 / 3 \mathrm{PL}$ selectivity remained persistent over time. A corresponding clustering analysis showed that alpha ERD evolved from sensorimotor areas to other cortical sites.

Inspection of the early responses in an across-subjects analysis revealed that responses to motion obeying the $2 / 3 \mathrm{PL}$ in both alpha and beta bands were more widespread than for motion obeying other laws. Across all motion types, the early alpha activity was more widespread than the early beta activity, which was focused at central motor sites before it spread to a wider area.

Together, our results reveal that ERD within both frequency bands was consistently stronger, more widespread, and arose faster during the observation of motion obeying the $2 / 3 \mathrm{PL}$ than motion with kinematic profiles obeying other power laws.

\section{The organization of kinematically driven alpha and beta ERD} The ERD in a motor ROI $(\mathrm{Cz}, \mathrm{FCz}, \mathrm{CPz})$ was strongest for the $2 / 3$ PL motion type in both alpha and beta bands. Significant alpha band ERD patterns were evoked by the 2/3 PL motion in the $\mathrm{Cz}$ electrode. In the beta band, ERD was significant in the more anterior $\mathrm{FCz}$ electrode. The power attenuations described here spatially match the increased BOLD activity reported by Dayan et al. (2007) in both the motor and SMA cortices when observing a similar abstract visual stimulus moving according to the $2 / 3 \mathrm{PL}$.

ERD was maximal for the $2 / 3$ PL motion type, becoming weaker and less widespread as the movement diverged from this specific motion type (quantified by the deviation of the beta exponent from 1/3, see Eq. 4). This clear preference for the $2 / 3 \mathrm{PL}$, found both in this ERD analysis and earlier fMRI findings (Dayan et al., 2007), supports the existence of a privileged neural imprint of the 2/3 PL in the human brain. Dayan et al. (2007) also reported that areas associated with mirror neuron (MN) activity were more responsive to motion complying with the $2 / 3 \mathrm{PL}$, thus suggesting a possible link between $\mathrm{MN}$ activities and motion kinematics. Lestou et al. (2008) established the functional involvement of parietofrontal areas in the processing of movement kinematics. Our results strengthen this conclusion; several frontal and right prefrontal electrodes in regions associated with the $\mathrm{MN}$ showed preferential tuning of the beta band responses to the 
2/3 PL motion. This is also consistent with the findings of an action imitation study (Quandt et al., 2011), which indicated the involvement of frontal cortical MN activity, exhibited as greater desynchronization in frontal electrodes.

Across-subjects analysis of the ERD responses (0.5-1.5 s) revealed a more diffuse spatial distribution of the alpha ERD while the beta ERD was more confined to central sites, showing typical bilateral responses (e.g., Hari et al., 1998). Our results, which are consistent with previous studies (Crone et al., 1998; McFarland et al., 2000), suggest distinct roles for the diffuse alpha and focused beta ERDs during evoked motor activation.

Alpha oscillations are classically considered to reflect an "idling" cortical state that is interrupted by motor or sensory processes (Gastaut, 1952), resulting in alpha ERD. Beta ERD is seen in motor planning around movement onset (Alegre et al., 2003; Rubino et al., 2006; Tzagarakis et al., 2010). Our results can be interpreted as reflecting possible interruption of the resting state because the baseline activity was measured in our subjects while observing a static fixation cross, before stimulus onset. Since, however, the four motion types were randomly presented, it is highly unlikely that the differences in response to the different motion types reflect different levels of anticipation or preparation. Still, although no explicit preparation was expected to occur in our paradigm, given that biological movement may trigger an attentional orienting response (Shi et al., 2010), this may explain the immediate onset in response to the $2 / 3$ PL motion, observed here.

\section{ERD and motion kinematics}

Both the sensorimotor ERD and the 2/3 PL have been independently shown to play a key role in action-perception coupling; ERD arises during motion execution and observation (Hari et al., 1998; Cochin et al., 1999; Babiloni et al., 2002; Muthukumaraswamy and Johnson, 2004; Marshall and Meltzoff, 2011; Press et al., 2011), and the $2 / 3$ PL is associated with motor cortex activation while executing movement (Moran and Schwartz, 1999) and observing motion (Dayan et al., 2007; Casile et al., 2010). However, ERD modulation by pure kinematic regularities has not been explicitly studied before.

An MEG study (Press et al., 2011; see also fMRI study by Di Dio et al., 2013) in which humans observed four movement types, a human hand or dot moving with either biological-like or constant velocity profiles, showed that motor cortical activity is more strongly affected by movement kinematics than by body form. Our results achieved through presenting a cloud of dots moving strictly according to the $2 / 3 \mathrm{PL}$ show that kinematics is sufficient to elicit motor activity reflected by stronger central ERD. Importantly, these studies used rich biological (naturalistic) kinematics, whereas we presented a mathematically driven (pure) kinematic regularity.

An action observation study showed that the alpha and beta ERD responses in dancers viewing dance movements are stronger than in nondancers observing similar movements (Orgs et al., 2008). The reported onsets of the selective ERDs occurred $\sim 1-2$ $s$ after movement onset, as observed here. Therefore, dominance onsets appear to be kinematically driven rather than being driven by body form. Avanzini et al. (2012) also reported beta band modulation by cyclic and noncyclic movements of a human-like agent. Our results, however, demonstrated a link between kinematics and ERD modulation in response to abstract stimuli rather than a human agent stimulus. This suggests that biological body form is not required to generate ERD modulation during motion observation.

\section{Spatiotemporal dominance of the $2 / 3 \mathrm{PL}$}

Our analysis aimed to characterize the spatiotemporal signature of ERD. Previous studies examined either evoked responses (for review, see Pfurtscheller and Lopes da Silva, 1999) or averaged spectral densities across movement blocks (e.g., Oberman et al., 2005) but generally neglected to assess finer temporal aspects of ERD. By identifying the dominant motion type for each electrode and clustering all electrodes based on their temporal signatures, we could directly compare three key ERD characteristics: identity and dominance onset for a given motion type and the corresponding electrode clusters that carry a shared temporal signature.

A central motor cluster in the alpha band and additional nonmotor areas of beta activity were not only consistently sensitive to the 2/3 PL but also exhibited faster dominance onset $(\sim 1 s)$ than other motion types. Other, mostly nonmotor, electrodes, for which the dominant motion type was not the $2 / 3$ PL motion, showed delayed dominance onset $(>2 \mathrm{~s})$. Thus, 2/3 PL kinematics alone, in the absence of biological or bodily form, social, or high level context, can evoke selective responses in the prefrontal cortex.

Different alpha and beta ERD patterns emerged from the grand average versus the across-subjects analysis. The acrosssubjects analysis $(0.5-1.5 \mathrm{~s})$ showed a widespread alpha activity and a more focused beta ERD (confined to central sites; Fig. 4). On the other hand, the grand average analysis ( $9 \mathrm{~s}$ ) revealed a more widespread beta versus a more focused alpha 2/3 PL ERD (Fig. 5). Previous studies (Crone et al., 1998; McFarland et al., 2000), using a time window of $\sim 1 \mathrm{~s}$, reported a widespread alpha ERD and a focused beta ERD as in our across-subjects analysis. Our grand average analysis, however, revealed that these alpha and beta 2/3 PL ERD patterns are not necessarily sustained over time; beta ERD responses to the 2/3 PL may become more widespread, whereas the alpha 2/3 PL ERD becomes more focused. The different temporal scales in these two types of analysis emphasized different activity patterns: the grand average clustering analysis, assessing motion type response persistence, was performed over a $9 \mathrm{~s}$ time window, whereas the across-subjects analysis assessed response robustness, separately for each motion type, during a $1 \mathrm{~s}$ time window. Nevertheless, a clear 2/3 PL preference emerged in both these different analyses.

In conclusion, our results indicate that motion kinematics is reflected by ERD in the human brain. Visual representations of motion may be best tuned to motion kinematics as expressed by the $2 / 3$ PL. The power attenuation of different rhythms is commonly used in brain machine interface research, for example, to decode fine movement properties using noninvasive techniques. In particular, the possibility of inferring motion kinematics based on noninvasive neural signals may be of great importance for developing and improving existing brain machine interface technologies to be used for motor rehabilitation (Jerbi et al., 2011).

\section{References}

Abend W, Bizzi E, Morasso P (1982) Human arm trajectory formation. Brain 105:331-348. CrossRef Medline

Alegre M, Labarga A, Gurtubay IG, Iriarte J, Malanda A, Artieda J (2003) Movement-related changes in cortical oscillatory activity in ballistic, sustained and negative movements. Exp Brain Res 148:17-25. CrossRef Medline

Avanzini P, Fabbri-Destro M, Dalla Volta R, Daprati E, Rizzolatti G, Cantalupo G (2012) The dynamics of sensorimotor cortical oscillations during the observation of hand movements: an EEG study. PLoS One 7:e37534. CrossRef Medline

Babiloni C, Babiloni F, Carducci F, Cincotti F, Cocozza G, Del Percio C, 
Moretti DV, Rossini PM (2002) Human cortical electroencephalography (EEG) rhythms during the observation of simple aimless movements: a high-resolution EEG study. Neuroimage 17:559-572. CrossRef Medline

Bell AJ, Sejnowski TJ (1995) An information-maximization approach to blind separation and blind deconvolution. Neural Comput 7:1129-1159. CrossRef Medline

Bernstein N (1967) The coordination and regulation of movements. Oxford: Pergamon.

Bizzi E, Tresch MC, Saltiel P, d'Avella A (2000) New perspectives on spinal motor systems. Nat Rev Neurosci 1:101-108. CrossRef Medline

Casile A, Dayan E, Caggiano V, Hendler T, Flash T, Giese MA (2010) Neuronal encoding of human kinematic invariants during action observation. Cereb Cortex 20:1647-1655. CrossRef Medline

Cochin S, Barthelemy C, Roux S, Martineau J (1999) Observation and execution of movement: similarities demonstrated by quantified electroencephalography. Eur J Neurosci 11:1839-1842. CrossRef Medline

Costantini M, Galati G, Ferretti A, Caulo M, Tartaro A, Romani GL, Aglioti SM (2005) Neural systems underlying observation of humanly impossible movements: an fMRI study. Cereb Cortex 15:1761-1767. CrossRef Medline

Crone NE, Miglioretti DL, Gordon B, Sieracki JM, Wilson MT, Uematsu S, Lesser RP (1998) Functional mapping of human sensorimotor cortex with electrocorticographic spectral analysis. I. Alpha and beta eventrelated desynchronization. Brain 121:2271-2299. CrossRef Medline

Dayan E, Casile A, Levit-Binnun N, Giese MA, Hendler T, Flash T (2007) Neural representations of kinematic laws of motion: evidence for action-perception coupling. Proc Natl Acad Sci U S A 104:20582-20587. CrossRef Medline

Delorme A, Makeig S (2004) EEGLAB: an open source toolbox for analysis of single-trial EEG dynamics including independent component analysis. J Neurosci Methods 134:9-21. CrossRef Medline

de'Sperati C, Viviani P (1997) The relationship between curvature and velocity in two-dimensional smooth pursuit eye movements. J Neurosci 17:3932-3945. Medline

Di Dio C, Di Cesare G, Higuchi S, Roberts N, Vogt S, Rizzolatti G (2013) The neural correlates of velocity processing during the observation of a biological effector in the parietal and premotor cortex. Neuroimage 64: 425-436. CrossRef Medline

Fitts PM (1954) The information capacity of the human motor system in controlling the amplitude of movement. J Exp Psychol 47:381-391. CrossRef Medline

Flash T, Hochner B (2005) Motor primitives in vertebrates and invertebrates. Curr Opin Neurobiol 15:660 - 666. CrossRef Medline

Flash T, Hogan N (1985) The coordination of arm movements: an experimentally confirmed mathematical model. J Neurosci 5:1688-1703. Medline

Gangitano M, Mottaghy FM, Pascual-Leone A (2004) Modulation of premotor mirror neuron activity during observation of unpredictable grasping movements. Eur J Neurosci 20:2193-2202. CrossRef Medline

Gastaut H (1952) Etude électrocorticographique de la réactivité des rythmes rolandiques. Rev Neurol 87:176-182. Medline

Halliday DM, Rosenberg JR (1999) Time and frequency domain analysis of spike train and time series data. In: Modern techniques in neuroscience research, pp. 503-543. New York: Springer.

Hari R, Forss N, Avikainen S, Kirveskari E, Salenius S, Rizzolatti G (1998) Activation of human primary motor cortex during action observation: a neuromagnetic study. Proc Natl Acad Sci U S A 95:15061-15065. CrossRef Medline

Hatsopoulos NG (2009) Rhythms in motor processing: functional implications for motor behavior. Where do they come from and what are they good for? (short course). Boston: Society for Neuroscience Rhythms of the Neocortex.

Jasper H, Penfield W (1949) Electrocorticograms in man: effect of voluntary movement upon the electrical activity of the precentral gyrus. Eur Arch Psychiatry Clin Neurosci 183:163-174.

Jerbi K, Vidal JR, Mattout J, Maby E, Lecaignard F, Ossandon T, Hamamé CM, Dalal SS, Bouet R, Lachaux JP, Leahy RM, Baillet S, Garnero L, Delpuech C, Bertrand O (2011) Inferring hand movement kinematics from MEG, EEG and intracranial EEG: from brain-machine interfaces to motor rehabilitation. IRBM 32:8-18. CrossRef

Kühn AA, Doyle L, Pogosyan A, Yarrow K, Kupsch A, Schneider GH, Hariz MI, Trottenberg T, Brown P (2006) Modulation of beta oscillations in the subthalamic area during motor imagery in Parkinson's disease. Brain 129:695-706. CrossRef Medline

Lacquaniti F, Terzuolo C, Viviani P (1983) The law relating kinematic and figural aspects of drawing movements. Acta Psychol 54:115-130. CrossRef Medline

Leocani L, Comi G (2006) Movement-related event-related desynchronization in neuropsychiatric disorders. Prog Brain Res 159:351-366. CrossRef Medline

Lepage JF, Théoret H (2006) EEG evidence for the presence of an action observation-execution matching system in children. Eur J Neurosci 23: 2505-2510. CrossRef Medline

Lestou V, Pollick FE, Kourtzi Z (2008) Neural substrates for action understanding at different description levels in the human brain. J Cogn Neurosci 20:324-341. CrossRef Medline

Levit-Binnun N, Schechtman E, Flash T (2006) On the similarities between the perception and production of elliptical trajectories. Exp Brain Res 172:533-555. CrossRef Medline

Makeig S, Delorme A, Westerfield M, Jung TP, Townsend J, Courchesne E, Sejnowski TJ (2004) Electroencephalographic brain dynamics following manually responded visual targets. PLoS Biol 2:747-762. CrossRef Medline

Marshall PJ, Meltzoff AN (2011) Neural mirroring systems: exploring the EEG mu rhythm in human infancy. Dev Cogn Neurosci 1:110-123. CrossRef Medline

McFarland DJ, Miner LA, Vaughan TM, Wolpaw JR (2000) Mu and beta rhythm topographies during motor imagery and actual movements. Brain Topogr 12:177-186. CrossRef Medline

McGill R, Tukey JW, Larsen WA (1978) Variations of box plots. Am Statistician 32:12-16. CrossRef

Mognon A, Jovicich J, Bruzzone L, Buiatti M (2011) ADJUST: an automatic EEG artifact detector based on the joint use of spatial and temporal features. Psychophysiology 48:229-240. CrossRef Medline

Moran DW, Schwartz AB (1999) Motor cortical activity during drawing movements: population representation during spiral tracing. J Neurophysiol 82:2693-2704. Medline

Muthukumaraswamy SD, Johnson BW (2004) Primary motor cortex activation during action observation revealed by wavelet analysis of the EEG. Clin Neurophysiol 115:1760-1766. CrossRef Medline

Neuper C, Pfurtscheller G (2001a) Event-related dynamics of cortical rhythms: frequency-specific features and functional correlates. Int J Psychophysiol 43:41-58. CrossRef Medline

Neuper C, Pfurtscheller G (2001b) Evidence for distinct beta resonance frequencies in human EEG related to specific sensorimotor cortical areas. Clin Neurophysiol 112:2084-2097. CrossRef Medline

Nikouline VV, Linkenkaer-Hansen K, Wikström H, Kesäniemi M, Antonova EV, Ilmoniemi RJ, Huttunen J (2000) Dynamics of mu-rhythm suppression caused by median nerve stimulation: a magnetoencephalographic study in human subjects. Neurosci Lett 294:163-166. CrossRef Medline

Oberman LM, Hubbard EM, McCleery JP, Altschuler EL, Ramachandran VS, Pineda JA (2005) EEG evidence for mirror neuron dysfunction in autism spectrum disorders. Cogn Brain Res 24:190-198. CrossRef Medline

Orgs G, Dombrowski JH, Heil M, Jansen-Osmann P (2008) Expertise in dance modulates alpha/beta event-related desynchronization during action observation. Eur J Neurosci 27:3380-3384. CrossRef Medline

Pavlidou A, Schnitzler A, Lange J (2014) Distinct spatio-temporal profiles of beta-oscillations within visual and sensorimotor areas during action recognition as revealed by MEG. Cortex 54:106-116. CrossRef Medline

Perry A, Bentin S (2009) Mirror activity in the human brain while observing hand movements: a comparison between EEG desynchronization in the $\mu$-range and previous fMRI results. Brain Res 1282:126-132. CrossRef Medline

Pfurtscheller G (2001) Functional brain imaging based on ERD/ERS. Vision Res 41:1257-1260. CrossRef Medline

Pfurtscheller G, Lopes da Silva FH (1999) Event-related EEG/MEG synchronization and desynchronization: basic principles. Clin Neurophysiol 110:1842-1857. CrossRef Medline

Pfurtscheller G, Stancák A, Edlinger G (1997) On the existence of different types of central beta rhythms below $30 \mathrm{~Hz}$. Electroencephalogr Clin Neurophysiol 102:316-325. CrossRef Medline 
Pfurtscheller G, Brunner C, Schlögl A, Lopes da Silva FH (2006) Mu rhythm (de) synchronization and EEG single-trial classification of different motor imagery tasks. Neuroimage 31:153-159. CrossRef Medline

Press C, Cook J, Blakemore SJ, Kilner J (2011) Dynamic modulation of human motor activity when observing actions. J Neurosci 31:2792-2800. CrossRef Medline

Quandt LC, Marshall PJ, Bouquet CA, Young T, Shipley TF (2011) Experience with novel actions modulates frontal alpha EEG desynchronization. Neurosci Lett 499:37-41. CrossRef Medline

Richardson MJ, Flash T (2002) Comparing smooth arm movements with the two-thirds power law and the related segmented-control hypothesis. J Neurosci 22:8201-8211. Medline

Rubino D, Robbins KA, Hatsopoulos NG (2006) Propagating waves mediate information transfer in the motor cortex. Nat Neurosci 9:1549-1557. CrossRef Medline

Shi J, Weng X, He S, Jiang Y (2010) Biological motion cues trigger reflexive attentional orienting. Cognition 117:348-354. CrossRef Medline
Sibson R, Thomson GD (1981) A seamed quadratic element for contouring. Computer J 24:378-382. CrossRef

Soechting JF, Lacquaniti F (1981) Invariant characteristics of a pointing movement in man. J Neurosci 1:710-720. Medline

Talairach J, Tournoux P (1988) Co-planar stereotaxic atlas of the human brain. 3-dimensional proportional system: an approach to cerebral imaging. New York: Thieme.

Todorov E, Jordan MI (1998) Smoothness maximization along a predefined path accurately predicts the speed profiles of complex arm movements. J Neurophysiol 80:696-714. Medline

Tzagarakis C, Ince NF, Leuthold AC, Pellizzer G (2010) Beta-band activity during motor planning reflects response uncertainty. J Neurosci 30: 11270-11277. CrossRef Medline

Viviani P, Flash T (1995) Minimum-jerk, two-thirds power law, and isochrony: converging approaches to movement planning. J Exp Psychol Hum Percept Perform 21:32-53. CrossRef Medline

Viviani P, Stucchi N (1992) Biological movements look uniform: evidence of motor-perceptual interactions. J Exp Psychol 18:603. CrossRef Medline 